\title{
MAP-V Secucam Using Pixel to Pixel Matching Algorithm
}

\author{
Saritha Suvarna ${ }^{1}$, Manisha $K^{2}$, Pratheeksha ${ }^{3}$, R Priyanka ${ }^{4}$, Vinay Kumar ${ }^{5}$ \\ Assistant Professor, Department of CSE, Canara Engineering College, Benjanapadavu, Mangalore, India ${ }^{1}$
}

Research Scholar, Department of ISE, Canara Engineering College, Benjanapadavu, Mangalore, India ${ }^{2,3,4,5}$

\begin{abstract}
An intelligent monitoring device is an application which is developed for the security purpose and less storage convenience. The proposed system is applicable in any of the areas, which are needed to be monitored all the time using MAP-V Secucam. By combining the application and MAP-V Secucam we can use this system as an intelligent monitoring device. The MAP-V Secucam is used to catch the live images of the area in which it is being implemented, if any motion is detected, the MAP-V Secucam will be turned to recording mode. The recorded footages are stored in a particular folder. As the device detects the motion, it sends the alerts using web service. In this way the system will provide the security against any misdeed.
\end{abstract}

Keywords: Web surveillance system, Secucam, Security, Web Service.

\section{INTRODUCTION}

Security is one of the most important problems all over the world. This system basically concern on the use of automatic motion detection application using webcams for security purpose. Today in our country, security is one of the major issues and having a $24 * 7$ human eye is just impossible. In order to be secured of safety, it is useful to realize and manage smart surveillance system combined with image processing techniques[1].

Along with the security, storage is also considered important. So it is clever to reduce the amount of storage as much as possible. Storage of unnecessary data can be avoided and only the required data is to be stored. It eases the data access along with providing low cost storage.

Intelligent Monitoring device is an application in which MAP-V Secucam is used as a powerful motion detector. Instead of using various ultra-sonic or other sensors, we are using camera as the sensor here. By using the camera we can use this system to get alerted of any problem occurring and also have the proof for any misdeed. This can be used to keep track of the activities of the area, either of high security or low security. If any motion is detected the camera starts recording, stores them in system drive and the stored footage can be used further. Once the changes are being detected, the alert messages are sent to the receiver at the other end using web service.

There are many existing devices in market such as CCTV Cameras, IP camera, Infrared sensors, Laser Sensor etc. These will result in huge storage.

\section{A. CCTV}

Implementation of CCTV cameras is very costly and has drawbacks since it require constant monitoring of every activity which is not ease. Continuous manual visualization hampers the productivity and time. Criminals can penetrate into the CCTV system, thereby facilitating criminal acts.

\section{$B$. IP camera}

Implementation of IP camera is also very costly and not feasible. This system cause major problems as it becomes open to hackers via internet

\section{Infrared Sensor and Laser Sensor}

These devices are quite economic in comparison to above devices however they have some drawbacks too. These devices are difficult to install and rarely available. One of the major disadvantages of infrared sensors is the size required to provide good resolution to the signal. To overcome all these problems we use web camera.

\section{Web camera}

Implementation of web camera is known for their low manufacturing cost and their high flexibility. They have also become a source of security and privacy issues.

\section{LITERATURE SURVEY}

According to the papers referred, some of the drawbacks are found in previously used systems. In the paper "Cost effective IP camera for video survelliance" Ming -Jiang Yang[2] proposed the use of IP camera. This IPcamera are very costly and less secure. In 2014 Leighton Watter Kille and Martin Maximino[3] proposed the use of CCTV in the paper" The effect of CCTV on public safety, research roundup" in this the maintance CCTV is not so easy as it records continuous activities. In $2011 \mathrm{Z}$ I Chowdhury[4] 


\title{
International Journal of Innovative Research in Electrical, Electronics, Instrumentation and Control Engineering
}

NCAEE 2017

\author{
National Conference on Advances in Electrical Engineering \\ NMAM Institute of Technology, Nitte \\ Vol. 5, Special Issue 2, April 2017
}

proposed the use of infrared sensor in the paper" Design and implementation of photoelectric infrared sensor based security system" it is rarely available and does not provides good resolution[5].

\section{A. Requirement Analysis}

The requirement analysis process involves deriving the system requirements through the observation of the existing system. A user interacts with the software through the user interface. The implementation of the software is of least importance to the end user . Thus, any software designed should have a good user-interface Maintaining the Integrity of the Specifications.

\section{B. System Configuration}

The MAP-V secucam is intended to provide security to the area which is under its surveillance. It therefore requires two configurations which are given below:

1) Software Configuration

- Operating System : Windows -XP or higher

- Platform : .NET

- Programming Language: SQL, C\#

- SDK:VS 2010

- MS SQL Management Studio 2005/2008

- Connectivity: TCP/IP

- Web Service: Fast Alerts WS for sending SMS

2) Hardware Configuration

- Processor:133-MHzIntel Pentium-class processor.

- Hard Disk:5 GB recommended.

- Memory:1GB of RAM, 2GB recommended

- Display: Standard output display.

- Keyboard: Standard Qwerty Keyboard for Interface

- Mouse: Standard mouse with two buttons.

- Others: Web camera

\section{III.METHODOLOGY}

The Fig. 1 represents the Architecture of MAP-V Secucam.He re we make use of pixel to pixel matching algorithm. In this system, the secucam will be capturing the images at a particular interval of time. These images are compared on the basis of their pixels[6]. If any changes are found, it starts recording and sends the alert to the other end[7]. Based on the sensitivity level there are 3 cases: LOW, HIGH, MID.

- HIGH: Here the sensitivity level is very high. Even if the change is very minute it starts recording.

- MEDIUM: In this level the security will be less as compared to high level here it will sense human and objects.

- LOW: Here sensitivity level is very low. It will record only major changes.

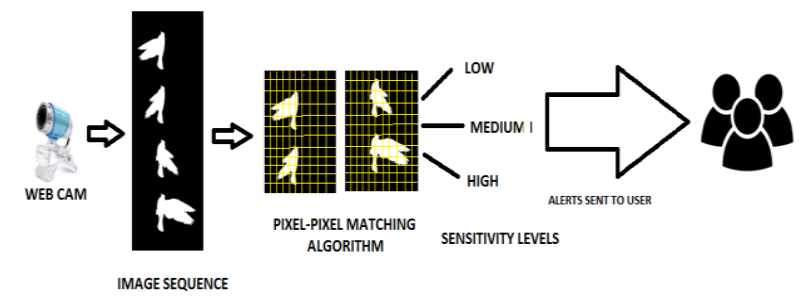

Fig. 1. MAP-V Scucam Architecture

A. Camera Interfacing

This form contains the list box where the list of all the camera devices connected to the software are displayed. By default the first camera is selected, but we can select any of the available devices from the listbox.After the device is selected, the camera is interfaced through coding part[8]. We can select the mode of our working and the application is set on.

Here we actually have three modes -

- High

- Middle

- Low

\section{B. Image Capturing}

The starts capturing images as per the time scheduled and in the intervals of the timer set. Images are captured in two frames which is then compared later.

\section{Motion Detection}

The basic working of motion detection module is comparison between two images. The first image is the one when we activate the application and the second image is of the second instant of time. When the application recognizes any changes between two corresponding images, it calls the next module i.e Image Capturing, and if not, it keeps monitoring again.

\section{Pixel to Pixel Matching Algorithm}

This module is used to compare the two frames captured, on the basis of their pixels. The percentage of the changes found is calculated. Then they are then classified as 3 sensitivity levels namely high, medium and low. Based on this we record the footage and send alert messages. It can be called as one of the motion detection algorithms

\section{IV.RESULT AND CONCLUSION}

MAP-V Secucam is security system which records the premises only when needed. User can effortlessly monitor home, office, a day. Time stamped image capturing let users capture details of events precisely. Different cradle, parking area, storehouse, UFO or any other premises 24hours environments have different surveillance requirements. Advances in PC based surveillance software now allow anyone with webcam to setup a robust, effective and inexpensive surveillance system. Today, all 
Vol. 5, Special Issue 2, April 2017

you need for securing your assets is a PC, a couple of webcams and software like Webcam based intelligent surveillance system. If any motion is detected in the camera it starts recording, stores them in the system drive than the stored footage can be used further. If the changes have been detected, the alert message is sent to the receiver at the other end using web service. This is achieved effectively, by minimizing the storage capacity.

\section{REFERENCES}

[1] Webcam Based Intelligent Surveillance System Akshada Deshmukh, Harshalata Wadaskar, Leena Zade, Neha Dhakate, Preetee Karmore.Published on march 2013

[2] Cost effective IP camera for video survelliance. Ming -Jiang Yang, Jo Yew Tham, Kwong Huang. Published on 2009

[3] The effect of CCTV on public safety, research roundup. Leighton Watter Kille and Martin Maximino. Published on 2014

[4] Design and implementation of photoelectric infrared sensor based security system. Z I chowdhury.Published on 2011

[5] Mohemed F Abdelkader, Integrated Motion Detection \& Tracking for Visual Surveillance (ICVS 2006)

[6] An improved motion detection method for Real-Time Surveillance Nan Lu,Jihang Wang,Q.H. Wu and Li Yang(19 February 2008).

[7] W.Hu,T.Tan,L.Wang,and S.Maybank.A survey on visual surveillance of obeject motion and behaviour, IEEE

[8] Implimentation of webcam based system for surveillance monitering, Bhramanandha Prabhu R,Arul Prabhar,Garima Bohra, Proceeding of ASCNT-2010,CDAC,Noida,India 